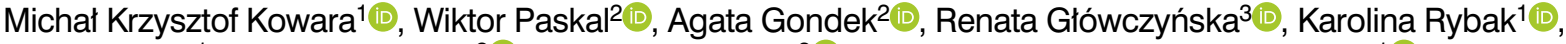
Maciej Kubik ${ }^{1}$, Paweł Włodarski² ${ }^{2}$, Grzegorz OpolskiiD, Agnieszka Cudnoch-Jędrzejewska ${ }^{1}$ (i)

${ }^{1}$ Chair and Department of Experimental and Clinical Physiology, Laboratory of Centre for Preclinical Research, Medical University of Warsaw, Warsaw, Poland

${ }^{2}$ Laboratory of Centre for Preclinical Research, Department of Methodology, Medical University of Warsaw, Warsaw, Poland

${ }^{3}$ First Department of Cardiology, Medical University of Warsaw, Poland.

\title{
Increased plasma levels of hsa-miR-21-5p in patients with reduced left ventricle ejection fraction admitted urgently due to acute coronary syndrome
}

\author{
Corresponding author: \\ Agnieszka Cudnoch-Jędrzejewska, \\ Chair and Department of Experimental \\ and Clinical Physiology, Laboratory \\ of Centre for Preclinical Research, \\ Medical University of Warsaw, Banacha \\ 1b, 02-097 Warsaw, Poland, \\ e-mail: agnieszka.cudnoch@wum.edu.pl \\ Medical Research Journal 2020; \\ Volume 5, Number 4, 250-255 \\ DOI: 10.5603/MRJ.a2020.0045 \\ Copyright (C) 2020 Via Medica \\ ISSN 2451-2591
}

\begin{abstract}
Myocardial ischaemia that occurs during acute coronary syndrome (ACS) induces a cascade of pathophysiological processes which might lead to left ventricle dysfunction reflected by decreased left ventricular ejection fraction (LVEF) in the echocardiographic examination. The enzymes regulating extracellular matrix (ECM) like matrix metalloproteinase 9 (MMP-9) also take part in this process. The MMP-9 activity is under control of a microRNA particle miR-21, which down-regulates its inhibitors. The study has shown, that in ACS population $(n=26)$ patients with LVEF $<50 \%$ presented increased miR-21 relative expression levels in plasma comparing to patients with LVEF $\geq 50 \%$ (3,33 vs. 1,64; $p=0,015)$. Moreover, a significant negative correlation between LVEF and miR-21 in this group of patients has also been presented.

Key words: acute coronary syndrome; metalloproteinase; extracellular matrix; micro-RNA; myocardial ischaemia; left ventricle ejection fraction
\end{abstract}

Med Res J 2020; 5 (4): 250-255

\section{Introduction}

Acute coronary syndromes (ACS) including unstable angina (UA), ST-Elevation Myocardial Infarction (STEMI) and Non-ST Elevation Myocardial Infarction (NSTEMI) have crucial epidemiological importance among heart diseases. The main reason for ACS is abrupt stenosis of coronary arteries mainly due to atherosclerotic plaque rupture and subsequent thrombosis which reduce the lumen of the vessel [1, 2]. As a result, myocardium undergoes ischaemia (like during UA) or even necrosis (like during NSTEMI and STEMI) [3]. Myocardial ischaemia leads to contractile dysfunction which can be observed on echocardiography as a reduction of left ventricle ejection fraction (LVEF) $[4,5]$. Various molecular pathways are activated and one of the released enzymes group, which causes structural changes of extracellular matrix constructing the scaffold for the entire heart muscle are matrix metalloproteinases $[6,7]$. Studies on mice model revealed that increase in expression of matrix metalloproteinase-9 (MMP-9) is observed during myocardial infarction and its plasma levels remain elevated from the first to the seventh day of the infarction. Induction of MMP-9 by LPS causes a decrease in LVEF in the course of myocardial infarction [8]. One of the factors regulating the activity of MMP-9 is a molecule of microRNA called hsa-miR-21-5p. MicroRNA are short (20-25 nucleotides), single-stranded RNAs synthesized endogenously which inhibit the expression of a specific mRNAs by the induction of their degradation through RISC complex [9]. Hsa-miR21-5p suppresses the expression of MMP-9 inhibitors (RECK and TIMP-3 proteins), thus contributing to the enhancement of MMP-9 activity $[10,11]$. In the present study, the authors used plasma from patients admitted to the hospital on emergency procedure due to ACS to compare the level of hsa-miR-21-5p expression in correlation with LVEF measured on echocardiography during hospitalization. 


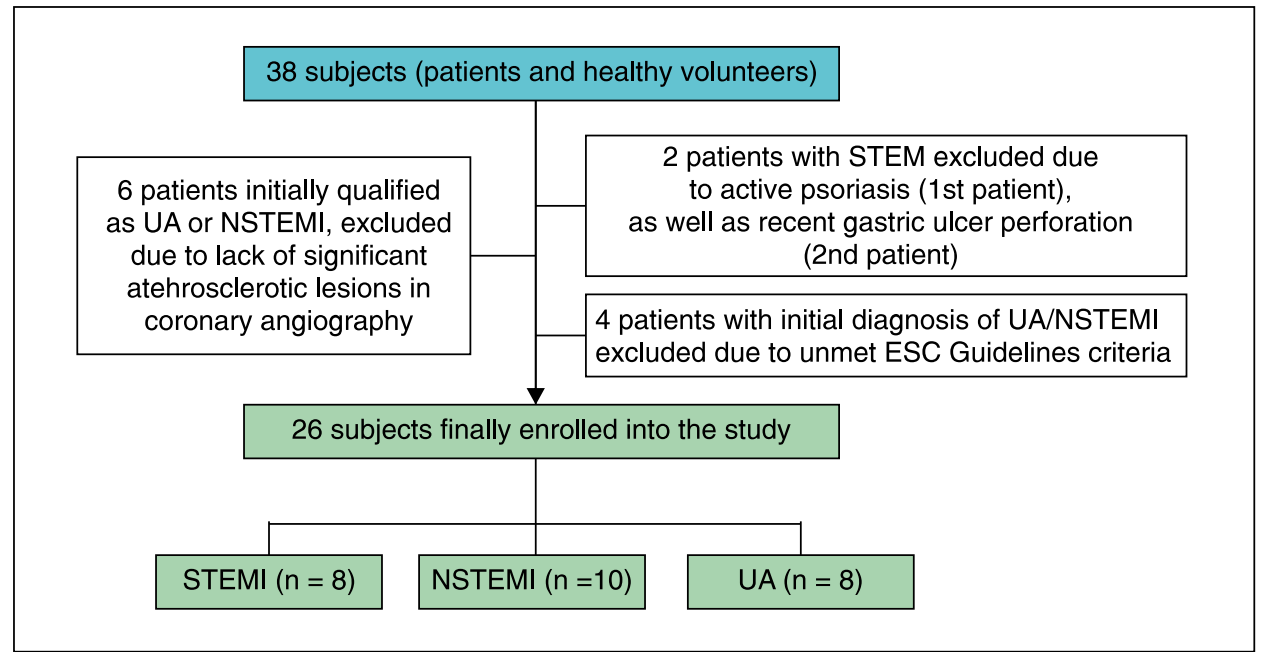

Figure 1. The enrolment to the study

\section{Materials and methods}

\section{The project and population of the study}

In this study, the plasma samples from patients who had been admitted to the hospital in an emergency procedure due to ACS were collected. The patients were recruited from October 2014 to March 2018. The study included patients with a definitively confirmed diagnosis of the acute coronary syndrome (UA, STEMI and NSTEMI) following guidelines of the European Society of Cardiology $[12,13]$. The excluding criteria were: - Malignant tumour (active or in the medical history),

- Active autoimmune or rheumatic disease,

- An operation or an invasive procedure for the 6 months before the ACS,

- Chronic Kidney Disease (grade G3b, G4 or G5 according to KDIGO, means eGFR $<45 \mathrm{ml} / \mathrm{min} / 1,73 \mathrm{~m} 2$ using MDRD formula),

- Diabetes mellitus (diagnosis according to guidelines of the American Diabetes Association - ADA),

- Acute infection or recovering from infectious disease during the last 3 months.

Initially, 38 patients were enrolled into this study but finally $n=26$ patients met all inclusion and exclusion criteria. All of the participants signed up with informed consent. The study obtained approval from the Bioethical Committee KB/55/2016 and KB/26/A/2017. Figure 1. shows the included protocol of the study.

\section{Blood samples}

Blood samples were collected from patients to EDTA tubes during the first 24 hours after the admission before coronary angiography with percutaneous coronary intervention. Samples were centrifuged (1200 x g, for
10 minutes), supernatants were aspirated and transferred to microcentrifuge tubes then placed in a freezer $\left(-80^{\circ} \mathrm{C}\right.$ temperature). The whole procedure from blood collection to the placement in a freezer lasted no longer than 4 hours. Additionally, blood samples were used to determine complete blood count, lipid profile, blood glucose level and creatinine concentration.

\section{Assay of hsa-miR-21-5p expression level via qRT- PCR method}

MiRNA molecules measured in plasma were: celmiR-39-3p („spike-in” control), hsa-miR-93-3p, hsamiR-191-5p (control miRNA) and researched molecule hsa-miR-21-5p. Plasma samples were placed in ice for a few minutes, then spined (20000 $\mathrm{xg}$ for 15 minutes in $\left.4^{\circ} \mathrm{C}\right)$. Clear supernatants $(300 \mu \mathrm{l})$ were transferred to the tubes "DNAse/RNAse free" and merged with denaturing buffer $(300 \mu \mathrm{l})$, then mixed by vortexing for 15 seconds. After 5 minutes of incubation, 30 femtomols of synthetic cel-miR-39-3p (index number: 10620000) phosphorylated in the 5' position was added to each sample as a „spike-in” control. Further isolation of miRNA was performed according to the producer guidelines (miRVana'M miRNA Isolation Kit, AM1560, Thermo Fisher Scientific). Expression levels of miRNA were assessed via „TaqMan Advanced miRNA Assays kit" (Thermo Fisher Scientific Co.) - cel-miR-39-3p (ID: 478293), hsa-miR-93-3p (ID: 478209), hsa-miR-191-5p (ID: 477952) and hsa-miR-21-5p (ID: 477975), according to the producer guidelines. Subsequently, RNA samples were succumbed to the reverse transcription using „TaqMan Advanced miRNA cDNA Synthesis Kit” (Thermo Fisher Scientific Co.). Finally, there were carried out qPCR in 96 holes plates using „Applied Biosystems 7500 Fast Real-Time PCR" system and 
Table 1. Baseline characteristics of the examined population, the difference between patients with ACS and LVEF $<50 \%$

\begin{tabular}{|c|c|c|c|}
\hline & $\begin{array}{c}\text { LVEF } \geq 50 \% \\
(n=14)\end{array}$ & $\begin{array}{c}\text { LVEF }<50 \% \\
(n=12)\end{array}$ & P (statistical significance) \\
\hline Age (years) ${ }^{1}$ & $62,2[9,2]$ & $63,1[14,2]$ & Insignificant* $(p=0,852)$ \\
\hline Sex, women (\%) & $2(14,3 \%)$ & $2(16,7 \%)$ & Insignificant ${ }^{+}(p=0,706)$ \\
\hline $\mathrm{BMI}\left(\mathrm{kg} / \mathrm{m}^{2}\right)^{1}$ & $27,7[4,1]$ & $27,1[4,1]$ & Insignificant * $(p=0,708)$ \\
\hline $\mathrm{TC}(\mathrm{mg} / \mathrm{dl})^{1}$ & $174,1[45,3]$ & $193,1[34,6]$ & Insignificant * $(p=0,291)$ \\
\hline $\operatorname{LDL}(\mathrm{mg} / \mathrm{dl})^{1}$ & $108,1[39,1]$ & $125,9[34,9]$ & Insignificant * $(p=0,313)$ \\
\hline $\mathrm{Hgb}(\mathrm{g} / \mathrm{dl})^{1}$ & $14,2[1,9]$ & $14,9[1,2]$ & Insignificant * $(p=0,261)$ \\
\hline Troponin I > MED², n (\%) & $5(35,7 \%)$ & $8(66,7 \%)$ & Insignificant ${ }^{+}(p=0,238)$ \\
\hline Hypertension, n (\%) & $8(57,1 \%)$ & $5(41,7 \%)$ & Insignificant ${ }^{+}(p=0,694)$ \\
\hline Active smoking, $\mathrm{n}(\%)$ & $8(57,1 \%)$ & $9(75 \%)$ & Insignificant $^{+}(p=0,589)$ \\
\hline Taking statins before ACS, n (\%) & $5(35,7 \%)$ & $3(25 \%)$ & Insignificant $^{+}(p=0,870)$ \\
\hline Myocardial infarction in medical history, $\mathrm{n}(\%)$ & $3(21,4 \%)$ & $2(16,7 \%)$ & Insignificant ${ }^{+}(p=0,848)$ \\
\hline
\end{tabular}

${ }^{1}$ results are presented as average [standard deviation]; ${ }^{2}$ number of patients with troponin I concentration higher than the median $(0,2625 \mathrm{ng} / \mathrm{ml})$ for all of the examined population on admission; *analysis performed using a t-Student test, ${ }^{+}$analysis performed using Yates's chi-squared test

„7500 Software V2.0.6” (Thermo Fisher Scientific Co.). Samples were assessed triply. Obtained $\mathrm{Ct}$ value (amount of PCR reaction cycles after detection threshold reached) of hsa-miR-21-5p, control hsamiR-93-3p and control hsa-miR-191-5p were referred to $C t$ value of cel-miR-39-3p -on this basis, there were calculated relative expression level of hsa-miR-21-5p using $2^{-\Delta \Delta \mathrm{Ct}}$ method.

\section{Echocardiography}

The echocardiograms were performed between 2nd and 5th day of hospitalisation in Echocardiography Laboratory of the 1st Department of Cardiology, University Clinical Centre, Medical University of Warsaw. The value of LVEF was measured using Simpson's method.

\section{Statistical analyses}

The character of a statistical distribution of data was evaluated using W-Shapiro-Wilk test. Qualitative and quantitative analyses for data of normal distribution were performed with Student's $t$-test (in case of homogenous variance) and Cochrane-Cox test (in case of heterogeneous variance) whereas the analyses of data presenting distribution different than normal were undertaken by Mann-Whitney $U$ test. The qualitative features were analysed via Yates's chi-squared test due to the total number of patients $\mathrm{n}<40$ while correlation analysis was performed using Spearman's Rank correlation coefficient. All analyses were conducted using STATISTICA 13 program, StatSoft Inc.

\section{Results}

\section{Characteristic of the general population}

According to LVEF value, the patients were divided into LVEF $\geq 50 \%$ group (preserved left ventricular ejection fraction, $n=14$ patients) and LVEF $<50 \%$ group (reduced left ventricular ejection fraction, $n=12$ patients). The median concentration of the troponin I in the entire population was $0,2625 \mathrm{ng} / \mathrm{ml}$. In Table 1. parameters are characterising the population included in this study, taking into account the differences between the two groups.

In the LVEF < 50\% group there were more patients with troponin I concentration over median value than in the whole population $(66,7 \%$ against to $35,7 \%$ in LVEF $\geq 50 \%$ group) but this difference wasn't statistically significant. No relevant differences were detected in the other parameters.

Of the total population of 26 people, 18 patients (approx. 70\%) were treated with primary percutaneous coronary intervention, 6 patients underwent coronary artery bypass grafting (CABG) whereas 2 patients were treated conservatively (optimal conservative treatment, without invasive treatment and fibrinolysis).

\section{The relative expression level of hsa-miR-21-5p in patients with ACS depending on the LVEF group}

It was demonstrated that the patients with ACS which had been classified into LVEF $<50 \%$ group presented significantly higher expression level of plasma hsa-miR-21-5p comparing to the patients classified into LVEF $\geq 50 \%$ group $(3,33[2,47-8,05]$ vs. $1,64[1,29-3,43], p=0,015)$ (Fig. 2.). 
The increase in relative expression level of hsamiR-21-5p in patients with LVEF $<50 \%$ compared to LVEF $\geq 50 \%$ subjects was also demonstrated in the subpopulation of NSTE-ACS patients (UA and NSTEMI, without STEMI, $\mathrm{n}=18)$ - the difference proved to be statistically significant $(1,61[1,29-1,98]$ vs. $3,17[2,27-$ $4,68], p=0,027)$. However, considering only the patients with myocardial infarction (according to the universal definition of myocardial infarction, $n=18$ ) — there was only a trend without statistical significance

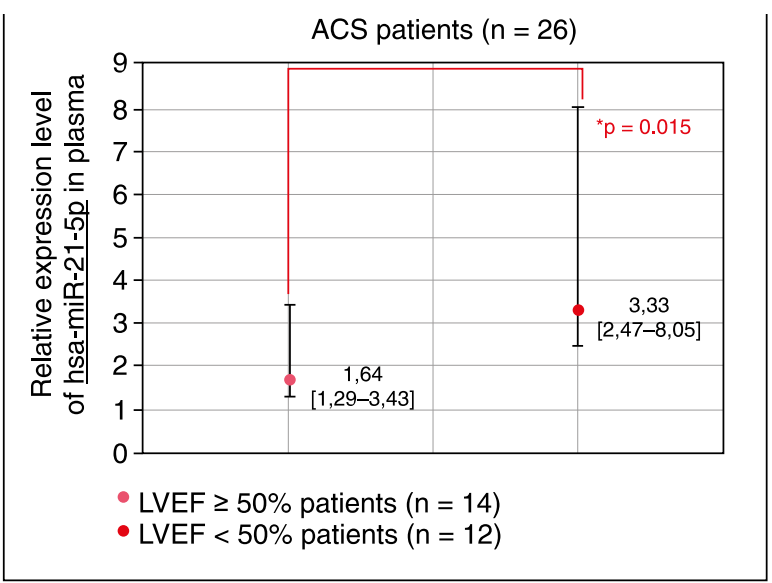

Figure 2. The difference in the relative expression level of hsa-miR-21-5p in patients with ACS with LVEF $<50 \%$ and LVEF $\geq 50 \%$. Results are presented using the median [interquartile range]. The Mann-Whitney $U$ test was performed for determination of statistical significance; *statistical significance $p<0,05$
$(3,49[3,07-11,42]$ vs. $1,67[1,36-6,11] ; p=0,077)$ in hsa-miR-21-5p relative expression level (Fig. 3.).

\section{The relative expression level of hsa-miR-21-5p} depending on LVEF value in a patient with ACS

An analysis of the correlation of hsa-miR-21-5p relative expression levels with the LVEF values in per cent was also performed - Spearman's Rank correlation coefficient is $\rho_{\mathrm{n}}=-0,44$; the result proved to be statistically significant $(p=0,024)$. The graph in Figure 4. demonstrates the correlation.

\section{Discussion}

Micro-RNA particles, including miR-21-5p, are currently being investigated as diagnostic and prognostic biomarkers of the acute coronary syndrome [14-16]. This study showed a correlation between a decrease in left ventricular ejection fraction and an increase in the expression level of the hsa-miR-21-5p particle in the serum of patients with the acute coronary syndrome. Patients with LVEF $<50 \%$ (assessed between $2^{\text {nd }}$ and $5^{\text {th }}$ day of hospitalization) have an increased level of hsa-miR-21-5p expression in plasma measured within the first 24 hours after admission. The hsa-miR-21-5p particle, although it is known as the "oncomir" (i.e. oncogenesis promotor, e.g. by increasing of anti-apoptotic $\mathrm{Bcl} 2$ expression), it also regulates the structure of extracellular matrix by suppressing the expression of MMP-9 inhibitors and thus enhancing the activity of this metalloproteinase $[17,18]$. Darabi et al.
A

NSTE-ACS patients $(n=18)$

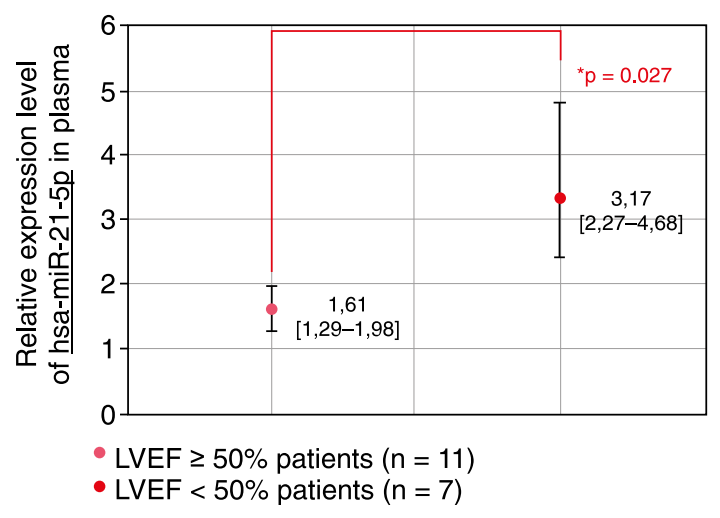

B

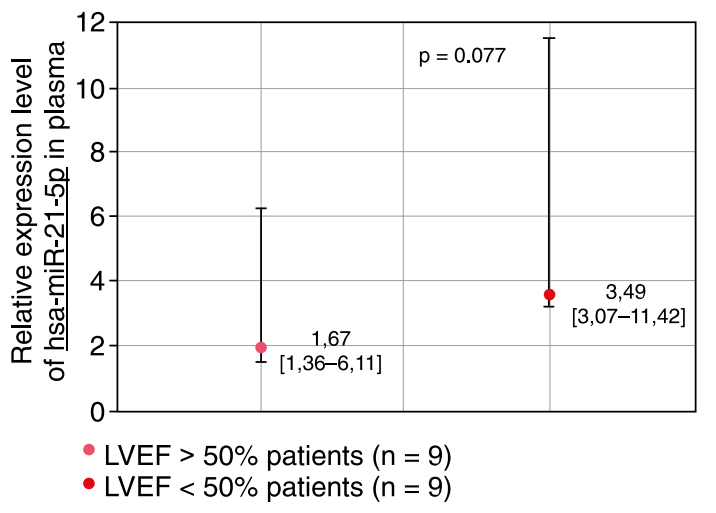

Figure 3. The difference in the relative expression level of hsa-miR-21-5p in patients with ACS with LVEF $<50 \%$ and LVEF $\geq 50 \%$ including only patients with NSTE-ACS (A) and myocardial infarction (MI) (B). Results are presented using the median [interquartile range]. The Mann-Whitney $U$ test was performed for determination of statistical significance; *statistical significance $p<0,05$ 


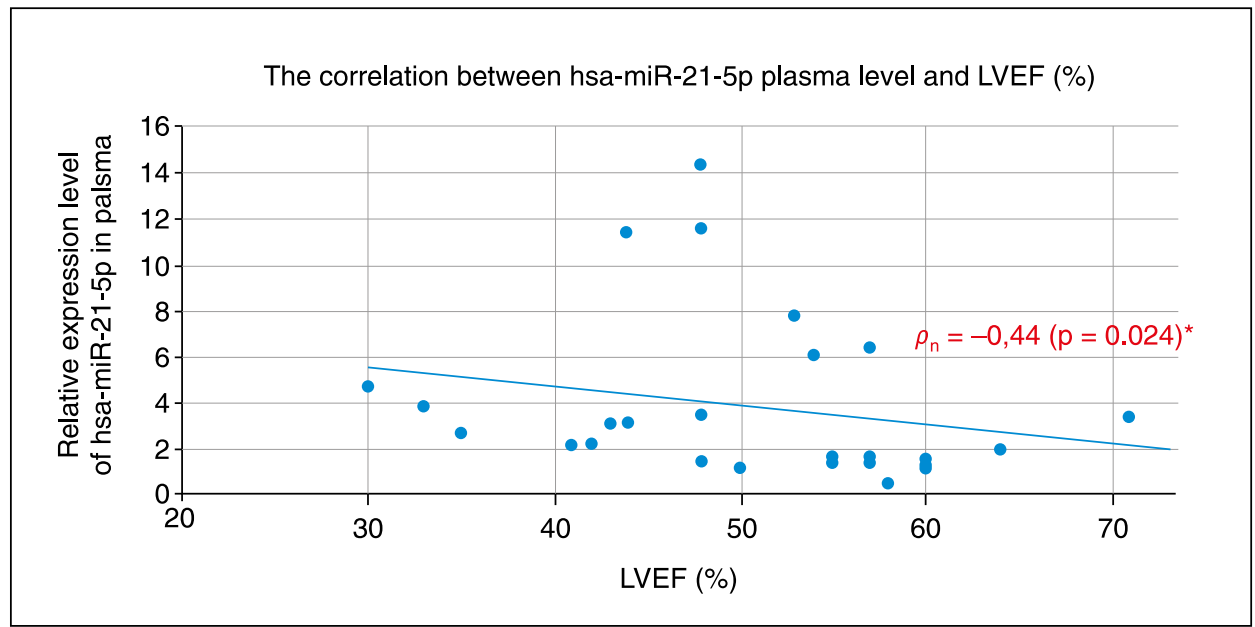

Figure 4. The correlation between hsa-miR-21-5p and LVEF (\%) values (\%). $\rho_{\mathrm{n}}$ - Spearman's Rank correlation coefficient; ${ }^{*} p<0,05$ considered as statistically significant

showed that the level of hsa-miR-21-5p expression is significantly increased in patients with acute coronary syndrome compared to patients with stable coronary disease (similar to the concentration of MMP-9 in the serum), while the presented study showed significantly increased expression of this particle in the group of patients with STEMI compared to both patients with UA and patients with stable coronary artery disease [19, 20]. The studies mentioned above indicate an increase in hsa-miR-21-5p expression in response to cardiomyocyte ischaemia, which may be associated with the activation of processes regulating the structure of the extracellular matrix in course of acute coronary syndrome. Liu et al. also showed that increased concentration of hsa-miR-21-5p measured on the 5th day of myocardial infarction is a predictor of post-infarction heart remodelling, which is an unfavourable process leading to impaired cardiac pump function [21]. Because the increased concentration of MMP-9 in the plasma of patients also correlates with markers of left ventricular dysfunction after myocardial infarction it can be hypothesized, that increased level of hsa-miR-21-5p, a positive regulator of MMP-9, is associated with increased damage due to myocardial ischaemia [22]. In addition, increased plasma levels of this microRNA particle have also been observed in patients with heart failure [23]. However, many studies showed a protective role of hsa-miR-21-5p up-regulation in acute coronary syndrome patients. The hsa-miR-21-5p particle, via its target gene PDCD4, is involved in cardiomyocyte preconditioning, limitation of the apoptosis of cells exposed to hypoxia and subsequent reoxygenation (ischaemia-reperfusion) in vitro and in vivo $[18,24]$. Moreover, it was observed that miR-21 demonstrates a cardioprotective role in ischaemic postconditioning through the regulation of PTEN / Akt pathway [25]. The ischaemia-induced activity of miR-21 stimulates production of cardioprotective molecules such as eNOS, HSP70 and HSF-1, which is a transcription factor for HSP70 [26]. Positive effects were also achieved by exogenous administration of the miR-21 particle to mice, which significantly reduced cardiomyocyte apoptosis and ischaemic necrosis. Moreover, it improved cardiac function by limiting the post-infarction decrease in the ejection and shortening fraction [27, 28]. Noteworthy, nanoparticles containing miR21 stimulate changes in the phenotype of macrophages localized in the area of ischaemic necrosis from pro-inflammatory to anti-inflammatory, which promote angiogenesis, tissue repair, reduce hypertrophy and fibrosis [29].

\section{Study limitations}

The limitation of the present study is a relatively small number of patients, which made the multivariate analysis impossible. For the same reason, it was difficult to demonstrate statistical significance, especially between more homogeneous groups of patients within the studied population.

\section{Summary}

In conclusion, the presented study showed a correlation between the expression level of the hsa-miR-21-5p particle in the plasma of patients with acute coronary syndrome and the reduction in left ventricular ejection fraction observed during hospitalization. Determination of the exact relationship between these variables and finding an answer to the question whether the increase in hsa-miR-21-5p expression in the acute phase of myocardial infarction is a beneficial, protective or aggravating, damaging factor require further research. 


\section{References}

1. Saleh M, Ambrose J. Understanding myocardial infarction. F1000Research. 2018; 7: 1378, doi: 10.12688/f1000research.15096.1.

2. Bob-Manuel T, Ifedili I, Reed G, et al. Non-ST Elevation Acute Coronary Syndromes: A Comprehensive Review. Curr Probl Cardiol. 2017; 42(9): 266305, doi: 10.1016/j.cpcardiol.2017.04.006, indexed in Pubmed: 28764841

3. James TN. The variable morphological coexistence of apoptosis and necrosis in human myocardial infarction: significance for understanding its pathogenesis, clinical course, diagnosis and prognosis. Coron Artery Dis. 1998; 9(5): 291-307, doi: 10.1097/00019501-19980905000007, indexed in Pubmed: 9710689.

4. Meybohm, P., , Assessment of left ventricular systolic function during acute myocardial ischemia: a comparison of transpulmonary thermodilution and transesophageal echocardiography. Minerva Anestesiol, 2011. 77(2): p. : 132-41

5. Grandmougin, D., , Development of a porcine beating-heart model of self-myocardial retroperfusion: evaluation of hemodynamic and cardiac responses to ischemia and clinical applications. J Cardiovasc Surg (Torino), 2018. 59(3): p. : 438-452.

6. Frangogiannis NG. Pathophysiology of Myocardial Infarction. Compr Physiol. 2015; 5(4): 1841-1875, doi: 10.1002/cphy.c150006, indexed in Pubmed: 26426469.

7. DeLeon-Pennell KY, Meschiari CA, Jung M, et al. Matrix Metalloproteinases in Myocardial Infarction and Heart Failure. Prog Mol Biol Trans Sci. 2017; 147: 75-100, doi: 10.1016/bs.pmbts.2017.02.001, indexed in Pubmed: 28413032.

8. DeLeon-Pennell K, Brás Ld, lyer R, et al. P. gingivalis lipopolysaccharide intensifies inflammation post-myocardial infarction through matrix metalloproteinase-9. Journal of Molecular and Cellular Cardiology. 2014; 76: 218-226, doi: 10.1016/j.yjmcc.2014.09.007.

9. Bartel D. MicroRNAs: Genomics, Biogenesis, Mechanism, and Function. Cell. 2004; 116(2): 281-297, doi: 10.1016/s0092-8674(04)00045-5

10. Fan $X$, Wang $E$, Wang $X$, et al. MicroRNA-21 is a unique signature associated with coronary plaque instability in humans by regulating matrix metalloproteinase-9 via reversion-inducing cysteine-rich protein with Kazal motifs. Exp Mol Pathol. 2014; 96(2): 242-249, doi: 10.1016/j. yexmp.2014.02.009, indexed in Pubmed: 24594117.

11. Dai J, Chen W, Lin Y, et al. Exposure to Concentrated Ambient Fine Particulate Matter Induces Vascular Endothelial Dysfunction via miR-21. Int J Biol Sci. 2017; 13(7): 868-877, doi: 10.7150/ijbs.19868, indexed in Pubmed: 28808419.

12. Roffi M, Patrono C, Collet JP, et al. ESC Scientific Document Group 2015 ESC Guidelines for the management of acute coronary syndromes in patients presenting without persistent ST-segment elevation: Task Force for the Management of Acute Coronary Syndromes in Patients Presenting without Persistent ST-Segment Elevation of the European Society of Cardiology (ESC). Eur Heart J. 2016; 37(3): 267-315, doi: 10.1093/eurheartj/ehv320, indexed in Pubmed: 26320110

13. Steg PhG, James SK, Atar D, et al. Task Force on the management of ST-segment elevation acute myocardial infarction of the European Society of Cardiology (ESC). ESC Guidelines for the management of acute myocardial infarction in patients presenting with ST-segment elevation. Eur Heart J. 2012; 33(20): 2569-2619, doi: 10.1093/eurheartj/ehs215, indexed in Pubmed: 22922416.

14. Zhou SS, Jin JP, Wang JQ, et al. miRNAS in cardiovascular diseases: potential biomarkers, therapeutic targets and challenges. Acta Phar- macol Sin. 2018; 39(7): 1073-1084, doi: 10.1038/aps.2018.30, indexed in Pubmed: 29877320.

15. Schulte C, Barwari T, Joshi A, et al. Comparative Analysis of Circulating Noncoding RNAs Versus Protein Biomarkers in the Detection of Myocardial Injury. Circulation Research. 2019; 125(3): 328-340, doi: 10.1161/circresaha.119.314937.

16. Kwee LC, Neely ML, Grass E, et al. Associations of osteopontin and NT-proBNP with circulating miRNA levels in acute coronary syndrome. Physiol Genomics. 2019; 51(10): 506-515, doi: 10.1152/physiolgenomics.00033.2019, indexed in Pubmed: 31530226.

17. Zhang J, Ma J, Long $\mathrm{K}$, et al Overexpression of Exosomal Cardioprotective miRNAs Mitigates Hypoxia-Induced H9c2 Cells Apoptosis. Int J Mol Sci. 2017; 18(4), doi: 10.3390/ijms18040711, indexed in Pubmed: 28350318.

18. Jazbutyte $\mathrm{V}$, Thum T. MicroRNA-21: from cancer to cardiovascular disease. Curr Drug Targets. 2010; 11(8): 926-935, doi: 10.2174/138945010791591403, indexed in Pubmed: 20415649.

19. Darabi F, Aghaei M, Movahedian A, et al. The role of serum levels of microRNA-21 and matrix metalloproteinase- 9 in patients with acute coronary syndrome. Mol Cell Biochem. 2016; 422(1-2): 51-60, doi: 10.1007/s11010-016-2805-z, indexed in Pubmed: 27590242.

20. Kowara, M., , Increased serum microRNA21 levels reflect cardiac necrosis rather than plaque vulnerability in patients with acute coronary syndrome: a pilot study. Kardiol Pol, 2019. 77(11): p. : 1074-1077.

21. Liu X, Dong Y, Chen S, et al. Circulating MicroRNA-146a and MicroRNA-21 Predict Left Ventricular Remodeling after ST-Elevation Myocardial Infarction. Cardiology. 2015; 132(4): 233-241, doi: 10.1159/000437090, indexed in Pubmed: 26337652

22. Squire IB, Evans J, Ng LL, et al. Plasma MMP-9 and MMP-2 following acute myocardial infarction in man: correlation with echocardiographic and neurohumoral parameters of left ventricular dysfunction. J Card Fail. 2004; 10(4): 328-333, doi: 10.1016/j.cardfail.2003.11.003, indexed in Pubmed: 15309700.

23. Marques F Vizi D, Khammy O, et al. The transcardiac gradient of cardio-microRNAs in the failing heart. European Journal of Heart Failure. 2016; 18(8): 1000-1008, doi: 10.1002/ejhf.517.

24. Cheng Y, Zhu P, Yang J, et al. Ischaemic preconditioning-regulated miR-21 protects heart against ischaemia/reperfusion injury via anti-apoptosis through its target PDCD4. Cardiovasc Res. 2010; 87(3) 431-439, doi: 10.1093/cvr/cvq082, indexed in Pubmed: 20219857.

25. Tu $Y$, Wan $L$, Fan $Y$, et al. Ischemic postconditioning-mediated miRNA-21 protects against cardiac ischemia/reperfusion injury via PTEN/Akt pathway. PLoS One. 2013; 8(10): e75872, doi: 10.1371/journal.pone.0075872, indexed in Pubmed: 24098402.

26. Yin C, Salloum F, Kukreja R. A Novel Role of MicroRNA in Late Preconditioning. Circulation Research. 2009; 104(5): 572-575, doi: 10.1161/circresaha.108.193250.

27. Huang W, Tian SS, Hang PZ et al Combination of microRNA-21 and microRNA-146a Attenuates Cardiac Dysfunction and Apoptosis During Acute Myocardial Infarction in Mice. Mol Ther Nucleic Acids. 2016; 5: e296, doi: 10.1038/mtna.2016.12, indexed in Pubmed: 26978580.

28. Gu GL, Xu XL, Sun XT, et al. Cardioprotective Effect of MicroRNA-21 in Murine Myocardial Infarction. Cardiovascular Therapeutics. 2015 33(3): 109-117, doi: 10.1111/1755-5922.12118.

29. Bejerano T, Etzion $S$, Elyagon $S$, et al. Nanoparticle Delivery of miRNA-21 Mimic to Cardiac Macrophages Improves Myocardial Remodeling after Myocardial Infarction. Nano Lett. 2018; 18(9): 5885-5891, doi: 10.1021/acs.nanolett.8b02578, indexed in Pubmed: 30141949 